PAR/LPTHE/93-54

Published in Phys. Lett. B 321 (1994) 394.

November 1993

\title{
STRANGE PARTICLE FREEZE-OUT
}

\author{
Jean Letessier ${ }^{1}$, Johann Rafelski ${ }^{1,2}$ and Ahmed Tounsi ${ }^{1}$ \\ ${ }^{1}$ Laboratoire de Physique Théorique et Hautes Energies*, Paris \\ ${ }^{2}$ Department of Physics, University of Arizona, Tucson, AZ 85721
}

\begin{abstract}
We reconsider thermal conditions of the central fireball presumed to be the source of abundantly produced strange (anti-)baryons in $\mathrm{S} \rightarrow \mathrm{W}$ collisions at $200 \mathrm{GeV}$ A. We show that it is possible to completely fix the freeze-out temperature of strange particles in terms of the central rapidity kaon to Lambda particle abundance ratio at fixed, high transverse mass using a non-equilibrium hadronization model and the measured quark fugacities.
\end{abstract}

Kinetic strange particle production models [1] imply that abundant strangeness is suggestive of the quark-gluon plasma (QGP). Even more specific information about the nature of the dense matter formed in relativistic nuclear collisions can be obtained considering strange quark and anti-quark clusters, since they are more sensitive to the environment from which they emerge [2]. Recently, the relative abundances of strange and multi-strange baryons and anti-baryons where studied experimentally [3, 1. It It has been suggested that the observed particle abundances are in agreement with a picture of explosively disintegrating QGP fireball [6]. This postulate was supported by the observation that details of the produced particle multiplicity point to a high entropy primordial phase [7], and by a comprehensive analysis of the data which concluded that the hadron gas (HG) model cannot be brought into consistency with the experimental results [8].

On the other hand, the alternative has been discussed [9, 10, 11] that the early experimental results [3], are compatible with the simplest possible scenario of an equilibrium, no flow, HG fireball being the particle source at which time it was noted that it is possible to distinguish the QGP and HG phases using the entropy content of the fireball [9]. We will show here that the HG alternative is not tenable anymore, given the larger and more precise set of experimental strange particle data [4, 5] and that the modifications required

* Unité associée au CNRS UA 280,

Postal address: LPTHE Université PARIS 7, Tour 24, 5è ét., 2 Place Jussieu, F-75251 CEDEX 05. 
in order to describe the final state hadrons point in a unique way to the originally postulated direct (that is without re-equilibration) QGP disintegration. We further show that the kaon multiplicity measurement is able to fix the unknown temperature of strange particle freeze-out.

Implicit in the physical picture employed here (see Ref. [8] for more details) is the formation of a central and hot matter fireball in the nuclear collisions at $200 \mathrm{GeV} \mathrm{A}$. This reaction picture presumes that a fraction of energy and flavor (baryon number) content of the central rapidity region is able to thermalize; this does not imply that the number of particles reaches "equilibrium" abundance characterized by the statistical variables of the system: the temperature $T$ and the chemical fugacities $\lambda_{i}$ of the different conserved quark flavors $i=u, d, s$. We recall that there is only a slight asymmetry in the number of $u$ and $d$ quarks in the heavy nuclei used in experiments: it is thus convenient to introduce the quark fugacity $\lambda_{\mathrm{q}}^{2}=\lambda_{\mathrm{u}} \lambda_{\mathrm{d}}$ and to confine the asymmetry between the number of neutrons and protons to the parameter $\delta \lambda \leq 0.03$ with $\lambda_{\mathrm{d}} / \lambda_{\mathrm{u}}=(1+\delta \lambda)^{2}$ [8].

Analysis of the S-S [12 and S-W data [8] obtained at $200 \mathrm{GeV}$ A has shown that the strange quark fugacity $\lambda_{\mathrm{s}} \simeq 1$, which is not the case for the lower energy results [13]. We recall that the recently reported $\bar{\Omega} / \Omega$ result [5] has provided not only a consistency confirmation of the thermal model but the finding $\bar{\Omega} / \Omega=0.6 \pm 0.4$ which depends solely on $\lambda_{\mathrm{s}}$ is also independently an indication that $\lambda_{\mathrm{s}} \sim 1\left[\mathbb{8}\right.$. The finding $\lambda_{\mathrm{s}}=1$ is of particular importance as it is natural for a directly disintegrating QGP phase: in QGP the symmetry between the $s$ and $\bar{s}$ quarks is reflected naturally in this value of $\lambda_{\mathrm{s}}$, and only if no HG reequilibration ensues can this value be preserved in the final state particle abundances. This is the case since in the HG phase, whatever the equation of state, $\lambda_{\mathrm{S}}=1$ is an exceptional condition. At final baryon number (viz. $\lambda_{\mathrm{d}}, \lambda_{\mathrm{u}} \neq 1$ ) the strangeness conservation constraint requires that the number of strange and anti-strange valance quarks bound in final state hadrons are equal. This is in general incompatible with $\lambda_{\mathrm{s}}=1$ as is most easily seen noting that the number of strange quarks contained in strange baryons is not equal to the number of anti-strange quarks contained in strange anti-baryons - however, this asymmetry may be compensated at some temperature $T$ by a similar asymmetry in the kaon yields, hence non-trivial conditions may exist with $\lambda_{\mathrm{s}}=1$ in the HG phase.

We now turn to the characterization of the final hadronic state in terms of the statistical parameters. The most convenient way is to size up the phase space in terms of all hadronic resonances, but to allow for flexibility regarding relative particle abundances. Throughout, we shall use the Boltzmann approximation for particle spectra. The distribution of the final state strange particles can be obtained by considering the Laplace transform of the phase space density, which leads to partition function $\mathcal{Z}_{\mathrm{s}}$ as given out of chemical equilibrium:

$$
\begin{aligned}
\ln \mathcal{Z}_{\mathrm{s}}=\frac{V T^{3}}{2 \pi^{2}}\{ & \left(\lambda_{\mathrm{s}} \lambda_{\mathrm{q}}^{-1}+\lambda_{\mathrm{s}}^{-1} \lambda_{\mathrm{q}}\right) \gamma_{\mathrm{s}} C_{\mathrm{M}}^{\mathrm{s}} F_{K}+\left(\lambda_{\mathrm{s}} \lambda_{\mathrm{q}}^{2}+\lambda_{\mathrm{s}}^{-1} \lambda_{\mathrm{q}}^{-2}\right) \gamma_{\mathrm{s}} C_{\mathrm{B}}^{\mathrm{s}} F_{Y} \\
& \left.+\left(\lambda_{\mathrm{s}}^{2} \lambda_{\mathrm{q}}+\lambda_{\mathrm{s}}^{-2} \lambda_{\mathrm{q}}^{-1}\right) \gamma_{\mathrm{s}}^{2} C_{\mathrm{B}}^{\mathrm{s}} F_{\Xi}+\left(\lambda_{\mathrm{s}}^{3}+\lambda_{\mathrm{s}}^{-3}\right) \gamma_{\mathrm{s}}^{3} C_{\mathrm{B}}^{\mathrm{s}} F_{\Omega}\right\}
\end{aligned}
$$

where the kaon $(K)$, hyperon $(Y)$, cascade $(\Xi)$ and omega $(\Omega)$ degrees of freedom are included successively. In the resonance sums all known strange hadrons are counted - in particular 
we have included kaons up to $1.78 \mathrm{GeV}$, hyperons up to $1.94 \mathrm{GeV}$, cascades up to $1.95 \mathrm{GeV}$ and also the omega resonance at $2.25 \mathrm{GeV}$. The phase space factors $F_{i}$ of the strange particles are explicitly

$$
\begin{array}{ll}
F_{K}=\sum_{j} g_{K_{j}} W\left(m_{K_{j}} / T\right), & F_{Y}=\sum_{j} g_{Y_{j}} W\left(m_{Y_{j}} / T\right), \\
F_{\Xi}=\sum_{j} g_{\Xi_{j}} W\left(m_{\Xi_{j}} / T\right), & F_{\Omega}=\sum_{j} g_{\Omega_{j}} W\left(m_{\Omega_{j}} / T\right),
\end{array}
$$

with $W(x)=x^{2} K_{2}(x)$, where $K_{2}$ is the modified Bessel function, arising as an integral over the free Boltzmann particle phase space. Compared to the case of an equilibrated source, several novel features are added to allow for the possibility that the final state particles emerge rapidly from a source of quite different nature than HG:

1. the factor $\gamma_{\mathrm{s}}$ in Eq. 10 [6] allows us to consider the strange particles emerging away from absolute chemical equilibrium which corresponds to the value $\gamma_{\mathrm{s}}=1$. In general, if there is not sufficient time to make strangeness (but sufficient time to exchange strange quarks between the carriers, which we implicitly assumed above) the partition function applies with $\gamma_{\mathrm{s}}<1$. The value of the factor $\gamma_{\mathrm{s}}$ is determined by the dynamics of strangeness production. Its measurement is only possible in the comparison of abundances of hadrons comprising different numbers of strange (or anti-strange) quarks. The value $\gamma_{\mathrm{s}}=0.7 \pm 0.1$ [8] arising from the WA 85 results [3] is suggestive of QGP based strangeness production mechanisms.

2. the factors $C_{i}^{\mathrm{s}}$ control the abundance of strange mesons $i=\mathrm{M}$ and baryons $i=\mathrm{B}$ with reference to the equilibrium based expectations [8]. A priori, these parameters could vary from particle to particle but intuitively we can subsume that the processes which form mesons and baryons from some primordial source differ substantially only between (strange) mesons and baryons. Especially when direct hadronization of a QGP is considered, one should not expect to see both factors to be equal to each other or to be unity.

We require that the final state hadrons contain equal number of $s$ and $\bar{s}$ quarks. This can be related to the parameters present in Eq. 1 using:

$$
0=\langle s\rangle-\langle\bar{s}\rangle=\lambda_{\mathrm{s}} \frac{\partial}{\partial \lambda_{\mathrm{s}}} \ln \mathcal{Z}_{\mathrm{s}}
$$

This is an implicit equation relating $\lambda_{\mathrm{s}}$ with $\lambda_{\mathrm{q}}$ for each given $T$. We stress that while we use above a partition function to size up the phase space into which particles are emitted, this procedure does not even tacitly imply existence of an intermediate HG phase formed in the QGP hadronization, and it may well be that the final state particles are emitted directly into final asymptotic states from the QGP phase. Recall that such a reaction picture is favored by the occurrence of the experimental value $\lambda_{\mathrm{s}}=1$.

We solve the condition (3) for the $\lambda_{\mathrm{s}}=1$ case, and obtain:

$$
\mu_{\mathrm{B}}^{0}=3 T \cosh ^{-1}\left(R_{\mathrm{C}}^{\mathrm{s}} \frac{F_{\mathrm{K}}}{2 F_{\mathrm{Y}}}-\gamma_{\mathrm{s}} \frac{F_{\Xi}}{F_{Y}}\right)
$$


Figure 1: Strangeness conservation constraint in the $\mu_{\mathrm{B}}-T_{\mathrm{f}}$ plane. Solid line: $\lambda_{\mathrm{s}}=1$, dashed curve: $\lambda_{\mathrm{s}}=0.98$, dotted line: $\lambda_{\mathrm{s}}=1.08$ (all with $\left.R_{\mathrm{C}}^{\mathrm{s}}=1, \gamma_{\mathrm{s}}=0.7\right)$. Horizontal intersecting lines correspond to $\lambda_{\mathrm{q}}=1.48 \pm 0.05$. Hatched area is the region of agreement (within 1 s.d.) of the strangeness conservation condition with the observed values $\lambda_{\mathrm{s}}=1.03 \pm 0.05$ and $\lambda_{\mathrm{q}}=1.48 \pm 0.05$. The experimental cross is set at the maximal possible temperature, $T_{\mathrm{f}}=0.232 \pm 0.005 \mathrm{GeV}$, consistent with $m_{\perp^{-}}$ spectra. Here $R_{\mathrm{C}}^{\mathrm{s}}=1.45$ in order to assure strangeness conservation.

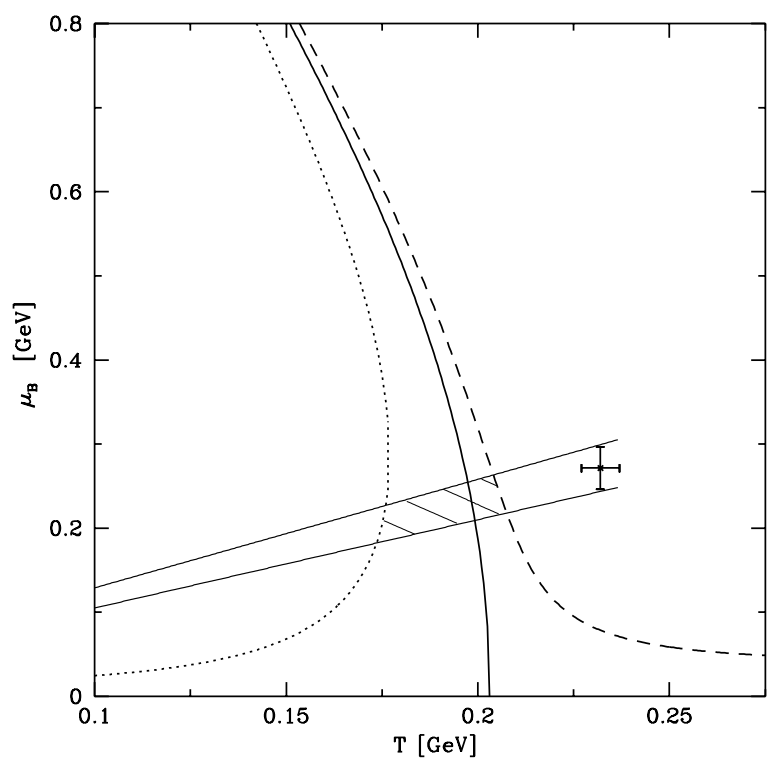

where we introduced the baryo-chemical potential $\mu_{\mathrm{B}}=3 T \ln \lambda_{\mathrm{q}}$ and the ratio

$$
R_{\mathrm{C}}^{\mathrm{s}}=\frac{C_{\mathrm{M}}^{\mathrm{s}}}{C_{\mathrm{B}}^{\mathrm{s}}} .
$$

We note that there is a real solution only when the argument on the right hand side in Eq. 1 is greater than unity. It turns out that this condition is a sensitive function of the temperature $T$ and of the hadronic resonances included in Eq.2. For any given hadronic spectrum used to compute the phase space factors $F_{i}$ there is a maximal temperature beyond which no such solution is possible. An important role is played by the factor $R_{\mathrm{C}}^{\mathrm{s}}$ of the dominant first term on the r.h.s. of Eq. 4 . The choice $R_{\mathrm{C}}^{\mathrm{s}}=1$ can be only justified for a source which is a well equilibrated HG. This hypothesis is inconsistent with $\gamma_{\mathrm{s}}=0.7$ and also challenged by the value $\lambda_{\mathrm{s}}=1$. More natural hypothesis is that the particles are emerging from rapidly disintegrating QGP phase. The study of the entropy content of the source points strongly in this direction as well [7].

But let us first consider the conservative case and take $R_{\mathrm{C}}^{\mathrm{s}}=1$, thus turn to the case of a HG source, and determine in how far can the strangeness conservation constraint can be reconciled with the full data sample of the experiment WA85 [3, 4, 5]. In Fig. 田 the solid line is just $\mu_{\mathrm{B}}\left(T ; R_{\mathrm{C}}^{\mathrm{s}}=1, \gamma_{\mathrm{s}}=0.7\right)$, see Eq. 4 . The dashed and, respectively, dotted curves are the solutions of the strangeness conservation condition for the experimentally permitted range of $\lambda_{\mathrm{s}}=0.98$ and, respectively, $=1.08$ (the mean experimental value is at $\lambda_{\mathrm{s}}=1.03$, [8]). The two dissecting curves correspond to the experimental constrain $\lambda_{\mathrm{q}}=1.48 \pm 0.05$. The hatched area is the region of $\mu_{\mathrm{B}}$ and $T$ compatible with a hadronic gas source in which meson to baryon abundance equilibrium is reached.

The $m_{\perp}$-spectra of the strange particles used in the multiplicity analysis indicate a common temperature $T=232 \pm 5 \mathrm{MeV}$, and this is the experimental cross, 3 s.d. to the right of the range found to be consistent with the experiment. Thus we can conclude that a 
strangeness neutral HG source (without flow) for strange particles, even allowing for offequilibrium strangeness saturation $\gamma_{\mathrm{s}}=0.7$, is in disagreement with experiment. It is at this point interesting to note that in the first analysis of the data [3] the "experimental" point coincided with the hatched area [9]. The more precise recent data [4] lead to a shift of the experimental point to the right by about 1 s.d. after a fit of the high statistics $m_{\perp}$ spectra; similarly the theory was also further developed and includes today all known hadronic resonances. This has moved the solid line $\left(\lambda_{\mathrm{s}}=1\right)$ in Fig. 1 by about 1 s.d. to the left, to its current location. Finally, the disagreement between HG-theory and experiment is enhanced by another 1 s.d. since previously we had $\lambda_{\mathrm{s}}>0.93$ (within 1 s.d.) and now it is $\lambda_{\mathrm{s}}>0.98$ (dashed line in Fig. 1), which moves the error boundary by one s.d. to the left, to its current location in Fig. 1. We conclude that these recent developments preclude the possibility of a HG interpretation of the data, without other dynamical features, such as transverse flow [8]. This conclusion seems to differ from the two-temperature hadronization model [14], which assumes a fully equilibrated (up to strangeness) sources of strange and non-strange particles at different temperatures, and does not seem to notice that the strange particle $m_{\perp}$-spectra are too hot for the HG-model temperatures.

To reach agreement today between the conditions of the source emitting the particles and strangeness conservation constraint we must allow for transverse surface flow. In presence of flow the thermal freeze-out temperature $T_{\mathrm{f}}$ of the particles would be blue-shifted according to the standard Doppler formula to the higher value $T_{\mathrm{E}}$ read off the $m_{\perp}$-spectra:

$$
T_{\mathrm{E}}=T_{\mathrm{f}} \sqrt{\frac{1+\beta_{\mathrm{f}}}{1-\beta_{\mathrm{f}}}} .
$$

Here $\beta_{\mathrm{f}}$ is the surface transverse velocity. The hatched area in Fig. 1 is then indicating a thermal freeze-out temperature $T_{\mathrm{f}}=190 \pm 15 \mathrm{MeV}$; such a high $T_{\mathrm{f}}$, though consistent with the notion of strangeness conservation (when one subsumes HG equilibrium meson and baryon abundances), cannot be physically correct, since it entails a much too high particle density. Should a QGP phase hadronize at this temperature, it must be expected that there would be continued hadronic particle interactions and therefore collective (transverse) flow of the resulting HG matter, with final particle decoupling occurring at lower temperatures, where density is low. Such a scenario involving HG final state evolution could not be expected to result in the $\lambda_{\mathrm{s}}=1$ which has been observed.

We believe that the value $\lambda_{\mathrm{s}} \simeq 1$ may be taken as suggesting a primordial phase hadronization in a manner in which the emerging hadrons immediately decouple from each other. One can imagine here two extreme cases: a freeze out at very low temperature, where the particle density is naturally low, or a sequential hadronization at high temperature, such that the produced hadronic particles are escaping freely. All this can in general be accomplished in consistency with strangeness conservation principle when allowing for the production of strange mesons and baryons away from abundances dictated by the relative HG chemical equilibrium. These putative scenarios are consistent with the diverse experimental facts and theoretical believes, but have a lot of freedom in the choice of values for the hadronization parameters. The issue is to find a physical observable which would allow 


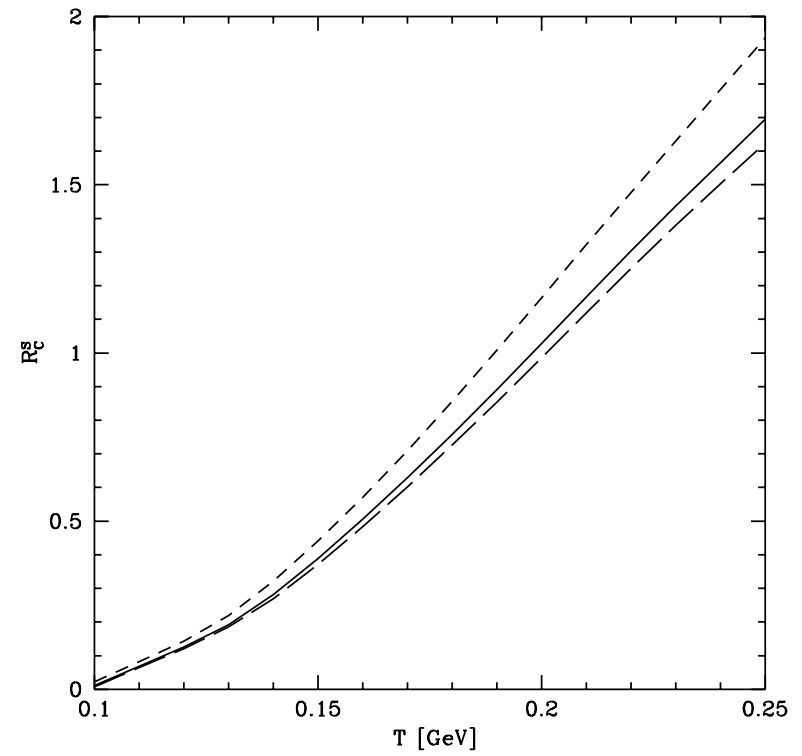

Figure 2: For each value of freeze-out temperature we need a different non-equilibrium parameter $R_{\mathrm{C}}^{\mathrm{s}}$. Solid line for $\lambda_{\mathrm{q}}=1.5$, longdashed line for $\lambda_{\mathrm{q}}=1.3$, short dashed curve $\lambda_{\mathrm{q}}=2$; all for $\lambda_{\mathrm{s}}=1, \gamma=0.7$.

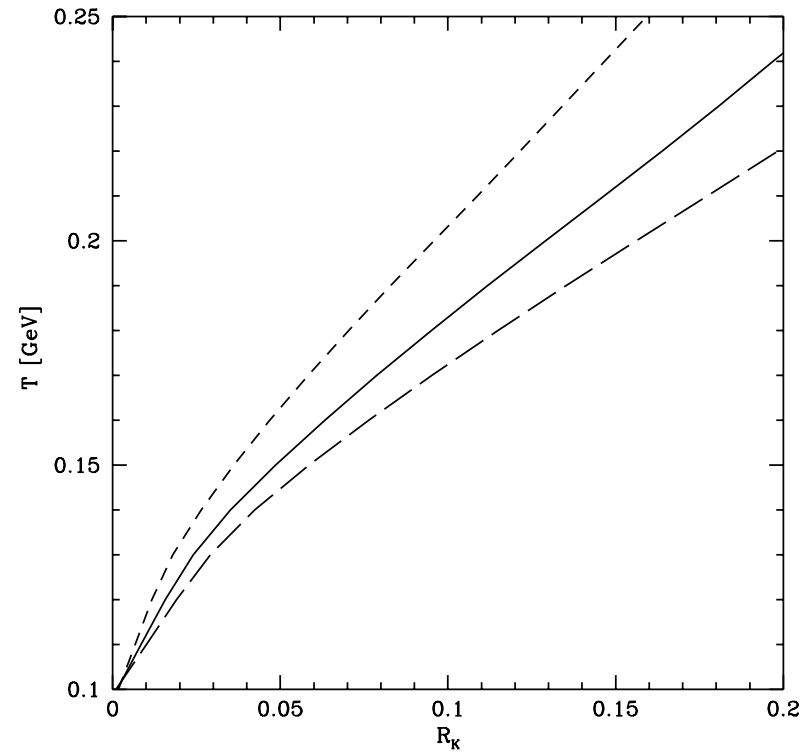

Figure 3: Each measured value of $R_{\mathrm{K}}$, the Kshort to Lambda ratio, implies a different value of the strange particle freeze-out temperature. Lines as in Fig. 2.

to restrict considerably the conditions at which strange particle hadronization occurs. This could be clearly accomplished if the temperature of the freeze-out were to be determined.

We first determine which values of $R_{\mathrm{C}}^{\mathrm{s}}$ are required to have strangeness conservation at a given thermal freeze-out temperature $T_{\mathrm{f}}$. We take the experimentally motivated $\gamma_{\mathrm{s}}=0.7$, though the deviation from unity is of little numerical importance in this argument. Since the (multi-)strange (anti-)baryon particle ratios normally fix $\lambda_{\mathrm{q}}$ and $\lambda_{\mathrm{s}}$ we take these as given: we continue to use the QGP freeze-out value $\lambda_{\mathrm{s}}=1$ and set $\lambda_{\mathrm{q}}$ to three values in Fig. 2: the solid line is for $\lambda_{\mathrm{q}}=1.5$, an appropriate choice for the case of $\mathrm{S}-\mathrm{W}$ collisions at $200 \mathrm{GeV} \mathrm{A}$, (when $\lambda_{\mathrm{s}}=1$ ), the long dashed line is for $\lambda_{\mathrm{q}}=1.3$ which we believe is nearly appropriate for the case of S-S collisions at $200 \mathrm{GeV}$ A [12] (it would lead to a central rapidity, high$m_{\perp}$ particle ratio $\left.\bar{\Lambda} / \Lambda=0.35\right)$. The short dashed curve is for the choice $\lambda_{\mathrm{q}}=2$ which is our rough guess for the case of $\mathrm{Pb}-\mathrm{Pb} 170 \mathrm{GeV}$ A collisions (and which would lead to $\bar{\Lambda} / \Lambda=0.063)$. We see that for this wide range of physical situations $(0.35 \leq \bar{\Lambda} / \Lambda \leq 0.063)$ we are finding rather similar values of $R_{\mathrm{C}}^{\mathrm{s}}$ for each given freeze-out temperature.

The most accessible physical observable which is sensitive to the parameter $R_{\mathrm{C}}^{\mathrm{s}}$, and which is insensitive to other parameters of the thermal model [8] is the ratio

$$
R_{\mathrm{K}} \equiv \frac{K_{\mathrm{s}}^{0}}{\Lambda+\Sigma^{0}}=\frac{R_{\mathrm{C}}^{\mathrm{s}}}{8} \frac{\lambda_{\mathrm{s}} / \lambda_{\mathrm{d}}+\lambda_{\mathrm{d}} / \lambda_{\mathrm{s}}}{\lambda_{\mathrm{s}} \lambda_{\mathrm{u}} \lambda_{\mathrm{d}}}
$$

where the second identity is only valid if resonance decay contributions largely cancel, which is the case [8]. For the rather light kaons there exist many different possibilities for secondary 
production through resonance decays, which limits somewhat the practical usefulness of this ratio, except at very high $m_{\perp}^{\text {cut }}>1.9 \mathrm{GeV}$. However, since the data of the experiment WA85 can be constrained to such large values of $m_{\perp}^{\text {cut }}$, we show in Fig. 返, how the freeze-out temperature $T_{\mathrm{f}}$ is determined by the measured ratio $R_{K}$ (it should be here kept in mind that along each line shown, for fixed $\lambda_{\mathrm{s}}=1, \lambda_{\mathrm{q}}=\{2,1.5,1.3\}$ the ratio $\bar{\Lambda} / \Lambda$ is given as stated above, once experimental results become available for $\mathrm{Pb}-\mathrm{Pb}$ case, a more appropriate value of $\lambda_{\mathrm{q}}$ may be considered).

The precise determination of the ratio $R_{\mathrm{K}}$ at high $m_{\perp}$ will in our opinion measure the hadronization temperature of the strange particles as is indicated in Fig. 3 , and in this way a full characterization of the thermal source is accomplished. For $\mathrm{S}-\mathrm{W}$ collisions, a value $R_{\mathrm{K}} \simeq$ 0.05 (at $m_{\perp}>1.9 \mathrm{GeV}$ would be consistent with the notion of hadronization/disintegration of the primordial (QGP) source at temperature of about $0.15 \mathrm{GeV}$. This leads to a value $R_{\mathrm{C}}^{\mathrm{s}} \simeq 0.4$, which implies, keeping the meson yield near the hadronic chemical equilibrium, that at this "low" temperature the hadronization process enriches by a factor 2.5 the naive thermal yield of strange baryons and anti-baryons. Should a considerably larger values of $R_{\mathrm{K}}$ (and hence also of $T_{\mathrm{f}}$ ) be found, it would suggest that the hadronization occurs by direct and very probably sequential evaporation of particles from the hot primordial (QGP) phase.

We have shown that in order to arrive at a satisfactory model of a low temperature rapidly hadronizing primordial phase we need to consider off-equilibrium strange particle abundances. We have proposed a method to measure the relevant parameter by determining precisely the central rapidity, high $m_{\perp}, \mathrm{K}_{s}$ to hyperon ratio. The sensitivity of this measurement is sufficient to allow to fix the freeze-out temperature of strange particles. We favor a low value at this time in order to have a consistent explanation of the finding $\lambda_{\mathrm{s}} \simeq 1$ in terms of a globally hadronizing state, with resulting particles escaping without forming an equilibrated HG phase. We await with interest the (precise) experimental results [15] which will settle in our opinion the choice of hadronization model and conditions.

Acknowledgement: J. R. acknowledges partial support by DOE, grant DE-FG0292ER40733 and thanks his co-authors for their kind hospitality in Paris.

\section{References}

[1] J. Rafelski, Phys. Rep. 88 (1982) 331.

J. Rafelski, in: Particle Production in Highly Excited Matter, H. H. Gutbrod and J. Rafelski (Eds.), Plenum, New York, 1993, p. 529.

[2] J. Rafelski and M. Danos, Phys. Lett. B192 (1987) 432.

[3] S. Abatzis et al., (WA85 collaboration) Phys. Lett. B259 (1991) 508;

S. Abatzis et al., (WA85 collaboration) Phys. Lett. B270 (1991) 123; 
[4] D. Evans, for the WA85 collaboration, New results from WA85 on multi-strange hyperon production in $200 \mathrm{~A} \mathrm{GeV/c} \mathrm{S-W} \mathrm{interactions,} \mathrm{presented} \mathrm{at} \mathrm{the} \mathrm{"Quark} \mathrm{Matter} \mathrm{'93"}$ meeting, June 1993, to appear in the proceedings (Nucl. Phys. A).

[5] S. Abatzis et al, (WA85 collaboration) Phys. Lett. B316 (1993) 615.

[6] J. Rafelski, Phys. Lett. B262 (1991) 333;

J. Rafelski, Nucl. Phys. A544 (1992) 279c, and references therein.

[7] J. Letessier, A. Tounsi, U. Heinz, J. Sollfrank and J. Rafelski, Phys. Rev. Lett. 70 (1993) 3530 .

[8] J. Letessier, A. Tounsi, U. Heinz, J. Sollfrank and J. Rafelski, Strangeness Conservation in hot nuclear fireballs, Paris preprint LPTHE/92-27R submitted to Phys. Rev. D

[9] J. Letessier, A. Tounsi and J. Rafelski, Phys. Lett. B292 (1992) 417

J. Letessier, A. Tounsi and J. Rafelski, in: Proceedings of the XXVI International High Energy Physics Conference, Dallas, Texas, August 6-12, 1992, J. R. Sanford (ed.), American Institute of Physics, New York, 1993, p. 983.

[10] J. Cleymans and H. Satz, Z. Phys. C57 (1993) 135.

[11] J. Cleymans, K. Redlich, H. Satz, and E. Suhonen, Z. Phys. C58 (1993) 347.

[12] J. Sollfrank, M. Gaździcki, U. Heinz, and J. Rafelski, Regensburg preprint TPR-93-14, Z. Physik $\mathbf{C}$, in press.

[13] J. Rafelski and M. Danos, Strangeness Flow Differences in Nuclear Collisions at 15 and 200 GeV A, submitted to Phys. Rev. Lett.

[14] K. Redlich, J. Cleymans, H. Satz and E. Suhonen, Hadronization of quark-gluon plasma Bielefeld preprint BI-TP 93/44, to appear in the proceedings of the "Quark Matter '93" meeting, June 1993 (Nucl. Phys. A).

[15] J. B. Kinson, WA85 Collaboration, Nucl. Phys. A544 (1992) 321c. (Preliminary values of the kaon to Lambda ratio can be read of the transverse $m_{\perp}$ distributions on pages 330-331c. We find $R_{\mathrm{K}}=0.15 \pm 0.1$ which is of course not sufficiently precise for this discussion.) 